\title{
Gestión de la Calidad y Diseño Específico de los Procesos de Admisión en el Sistema Universitario Español: Estudio de Caso en una Universidad Privada
}

\author{
Quality Management and Specific Design of the Admissions \\ Process in the Spanish University System: Case Study of a \\ Private University
}

\author{
José María Ortíz Lozano * \\ Antonio Rúa Vieites \\ Universidad Pontificia Comillas
}

\begin{abstract}
En el curso académico 2017/18 se presenta para las universidades españolas de naturaleza pública la primera oportunidad de diseñar de forma específica sus procesos de admisión de estudiantes de Grado. Con tal motivo surge el propósito de investigar si proceder en este sentido ayuda a promover la calidad de las universidades entre sus estudiantes. Tras la realización de un cuestionario sobre 467 estudiantes de una universidad privada con experiencia en el diseño específico de sus procesos de admisión, se han obtenido evidencias de que aquél puede ayudar a promover la satisfacción de los estudiantes, así como servir para mejorar la percepción en términos de reputación e imagen de la universidad que los estudiantes tienen sobre ella, incentivándose por ello, según el modelo EFQM, una mejora en la gestión de la calidad basada en la excelencia por parte de la organización al promover el concepto fundamental de excelencia "añadir valor para el cliente".
\end{abstract}

Descriptores: Gestión de la calidad, Admisión de estudiantes, Educación superior, Satisfacción de alumnos, EFQM.

In the academic year 2017/18 there is an opportunity for the public Spanish universities to design specific processes of undergraduate admissions. With this aim, we have investigated whether proceeding in this direction helps to promote the quality of universities for their students. Taking as reference the structure of the EFQM model, we have studied the impact on student satisfaction regarding how to determine the admission of students and improving their perception in terms of the reputation and image of the institution. Following the completion of a questionnaire on a sample of 467 students from a private university with proven experience in the specific design of the admissions process, we have obtained evidence that a specific design can promote student satisfaction as well as serve to enhance the perception in terms of reputation and the image of the university students have of it.

Keywords: Quality management, Student recruitment, Higher education, Student satisfaction, EFQM.

*Contacto: jmortiz@comillas.edu

ISSN: $1696-4713$

www.rinace.net/reice/

revistas.uam.es/reice
Recibido: $\quad 15$ de junio 2016

$1^{\text {a }}$ Evaluación: 28 de septiembre 2016

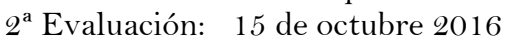

Aceptado: $\quad 7$ de noviembre 2016 


\section{Introducción}

En España, el Real Decreto 412/2014, de 6 de junio, que establece la normativa básica de los procedimientos de admisión a las enseñanzas universitarias oficiales de Grado recoge que "son las Universidades las que determinan, de conformidad con distintos criterios de valoración, la admisión a estas enseñanzas de aquellos estudiantes que hayan obtenido la titulación que da acceso a la universidad", especificando cuáles son esos posibles criterios de valoración según el tipo de acceso que pueden ser utilizados por las universidades. Éstos, a grandes rasgos, permiten la posibilidad de considerar, y aplicar desde el curso académico 2017/18, distintos criterios de valoración, entre los que se incluyen: 1, modalidad y materias cursadas en los estudios previos, equivalentes al título de Bachiller, en relación con la titulación elegida; 2, calificaciones obtenidas en materias concretas cursadas en los cursos equivalentes al Bachillerato español, o de la evaluación final de los cursos equivalentes al de Bachillerato español; 3, calificación final obtenida en las enseñanzas cursadas, y/o en módulos o materias concretas; 4, relación entre los currículos de las titulaciones anteriores y los títulos universitarios solicitados; 5, formación académica o profesional complementaria; estudios superiores cursados con anterioridad; o 6, establecer evaluaciones específicas de conocimientos y/o de competencias. En todo caso, la definición de distintos criterios de valoración en los procesos de admisión de las universidades españolas debe garantizar el respeto de los principios de igualdad, mérito y capacidad de los estudiantes.

Este escenario, por el que se permite a las universidades españolas establecer procesos específicos de admisión de estudiantes para estudios de Grado, es novedoso para las de naturaleza pública aunque no del todo para las de naturaleza privada. Sirva de ejemplo en este sentido, entre otros, las experiencias consolidadas de la Universidad de Navarra, Universidad de Deusto o la Universidad Pontificia Comillas (ICAI/ICADE), caso este último analizado en el presente trabajo. No obstante, es una realidad que las universidades españolas de naturaleza pública empiezan a plantearse, dadas las posibilidades que se abren a partir del curso académico 2017/18, la opción de desarrollar algún tipo de mecanismo específico para la selección de alumnos, como sucede, por ejemplo, con el título de Grado en Medicina de la Universidad Complutense de Madrid (Universidad Complutense de Madrid, 2015).

Por otro lado, es preciso señalar que tras la profunda reestructuración sufrida en los últimos años dentro del Sistema Universitario Español (SUE), como consecuencia del proceso de reconfiguración de los títulos universitarios españoles en su propósito de participar del proceso de creación y consolidación del Espacio Europeo de Educación Superior (EEES), se ha significado el interés creciente por dotar de sistemas de garantía de la calidad a los sistemas universitarios, constituyéndose como un pilar esencial del EEES (Feixas, 2004). Así, los problemas derivados de no proveer la calidad requerida son muchos e importantes, destacando entre ellos el impacto sobre la reputación e imagen de la institución, la dificultad para atraer talento académico, la penalización en rankings y los problemas con los que se pueden encontrar los egresados para ser contratados (Billing, 1996; Cruickshank, 2003).

Con estas premisas, dado que para la mayoría de las universidades españolas se presenta la primera oportunidad de diseñar específicamente sus procesos de admisión de estudiantes de Grado a partir del curso académico 2017/18, surge el propósito de investigar si proceder en este sentido ayuda a promover la calidad en las universidades 
españolas entre sus estudiantes, a partir del estudio de caso de una universidad privada con experiencia en el diseño de los referidos procesos.

\section{Antecedentes}

La aplicación de los principios y conceptos de la calidad en el ámbito universitario ha sido ampliamente recogida en la literatura académica, existiendo un mayoritario grado de consenso para asegurar la utilidad, beneficios y justificación del enfoque de una cultura de calidad en las universidades (Bandyopadhyay y Lichtman, 2007; Cruickshank, 2003; Harvey, 1998; Koch y Fisher, 1998; Marshall, 1998; Srikanthan y Dalrymple, 2002, 2005; Villarruel, 2010). En el ámbito universitario español, los sistemas de gestión de la calidad basados en la excelencia, o modelos de gestión de la excelencia se constituyen como una herramienta para la supervivencia en el largo plazo, permitiéndoles hacer frente a entornos cambiantes (Martín, 2005).

Cabe señalar también que las universidades españolas deben hacer frente en la actualidad a un escenario sumamente competitivo, especialmente las de naturaleza privada. Basta referir para ello el aumento de competencia experimentado en el sector en los últimos años; según datos del MECD, en el curso académico 2014-2015 el número total de universidades ascendía a 83, de las cuales 50 son de naturaleza pública y 33 privadas; destacando que en el periodo del año 2001 al 2014 se crearon 15 nuevas universidades, todas ellas de naturaleza privada (Ministerio de Educación, Cultura y Deporte, 2015). Tal y como afirma Vázquez García (2015):

La dinámica que se dibuja en el escenario universitario global es la de una mayor
presión de la competencia, guiada fundamentalmente por un mercado principal
basado en la reputación y el prestigio universitario, asentada en una mayor
diferenciación de las universidades"; siendo las principales consecuencias del citado
aumento de competencia el impacto en mejorar "la captación en número y calidad de
estudiantes, profesores e investigadores, en la intensificación de la carrera por la
excelencia y la atracción de talento, en la investigación y en el prestigio de la
institución. (p. 45)

$\mathrm{Al}$ aumento de competencia se unen las demandas realizadas por la sociedad para que las universidades españolas promuevan la calidad en todas las áreas de su actividad, como demuestra, por ejemplo, que la Ley Orgánica de Universidades (Ley Orgánica 6/2001, de 21 de diciembre), mediante la reforma que tiene lugar en 2007 por la Ley Orgánica 4/2007, de 12 de abril, aborde en su artículo 31 la necesidad de promover y garantizar la calidad de las universidades españolas, afirmando que ha de ser un fin esencial de la política universitaria.

Dentro de los modelos de gestión de excelencia aplicados en organizaciones europeas, sobresale la aplicación del modelo European Foundation for Quality Management (EFQM) de excelencia. Éste destaca por las capacidades que la literatura le reconoce dentro del campo de estudio de la gestión de la calidad en las organizaciones (Alfaro-Saiz et al., 2011; Campatelli, Citti y Meneghin, 2011; Lau y Anderson, 1998; Ruiz-Carrillo y Fernández-Ortiz, 2005; Sila y Ebrahimpour, 2002; Steed, 2002), donde destacan: 1) que facilita la implementación de los principios y conceptos de la gestión de la calidad al máximo nivel (Lau y Anderson, 1998), y 2) que permite evaluar la situación en la que una organización se encuentra en relación con los objetivos perseguidos de excelencia (Alfaro-Saiz et al., 2011). La utilidad del modelo EFQM también ha sido ampliamente contrastada en un contexto de organizaciones educativas (Alonso-Arévalo, 2003; Antón 
y Castilla, 2010; Calvo-Mora y Criado, 2003; Calvo-Mora, Leal y Roldan, 2005; Cantón y Vázquez, 2010; Martín, 2005; Ololube, 2006; Osseo-Asare y Longbottom, 2002; Rusjan, 2005; Steed, 2002; Zink y Schmidt, 1995) y se puede afirmar que ha tenido éxito a nivel mundial (Ololube, 2006).

Sin pretender profundizar en la estructura del modelo EFQM, se apuntan a continuación los principales elementos y características que lo definen. Según su propia definición, es un instrumento práctico y no prescriptivo sustentado en la promoción de los conceptos fundamentales de la excelencia en la organización (European Foundation for Quality Management, 2013).

Los conceptos fundamentales de excelencia que refiere el modelo EFQM son descritos como los principios requeridos para que cualquier organización obtenga la excelencia de forma sostenida, concretando, definiendo y profundizando los factores clave que a nivel general han sido establecidos por las organizaciones comprometidas con la calidad y la mejora continua. Los conceptos fundamentales de excelencia son ocho: 1) añadir valor para los clientes, 2) crear un futuro sostenible, 3) desarrollar la capacidad de la organización, 4) aprovechar la creatividad y la innovación, 5) liderar con visión, inspiración e integridad, 6) gestionar con agilidad, 7) alcanzar el éxito mediante el talento de las personas, y 8) mantener en el tiempo resultados sobresalientes.

El modelo EFQM provee de un marco de trabajo no prescriptivo que facilita la realización de los conceptos fundamentales de excelencia, con el beneficio añadido de permitir comprender las relaciones causa-efecto que existen entre lo que las organizaciones hacen y los resultados que obtienen. Se sustenta en nueve criterios donde están integrados los conceptos fundamentales de excelencia; cada criterio se divide en un número variable de subcriterios que representan aquello que normalmente tiene lugar en una organización excelente en referencia al criterio correspondiente. Los cinco primeros criterios (1: liderazgo, 2: estrategia, 3: personas, 4: alianzas y recursos, y 5: procesos, productos y servicios) se denominan agentes facilitadores y los cuatro restantes son resultados (6: resultados en clientes, 7: resultados en las personas, 8: resultados en la sociedad, y 9: resultados clave). Los agentes facilitadores tratan sobre lo que la organización logra y cómo lo logra, mientras que los resultados son consecuencia de los agentes facilitadores y de su análisis se deberán extraer las conclusiones necesarias para llevar a cabo los ajustes necesarios (European Foundation for Quality Management, 2013).

No obstante, conviene reparar en que la literatura también le reconoce limitaciones al modelo EFQM. Éstas derivan de su definición como marco de trabajo no prescriptivo, al no proveer de prácticas o líneas de actuación específicas que el management pueda considerar (Dahlgaard et al., 2013). Además, a pesar de que los modelos de gestión de excelencia proponen un marco de trabajo desde una perspectiva holística en su nivel conceptual, aquéllos adolecen de pautas para la integración en el nivel operacional (Dahlgaard-Park, 2008), siendo esta inconsistencia patente entre la definición de los criterios asociados a liderazgo y procesos (Dahlgaard et al., 2013), pues en el primero se consideran aspectos de la cultura de la organización tales como misión, visión, valores de la organización mientras que en el criterio procesos son obviados.

$\mathrm{Al}$ abordar el estudio de la gestión de la calidad en las universidades y su relación con la gestión de los procesos de admisión en las universidades, se ha confirmado que dicha relación apenas ha sido estudiada. Las escasas investigaciones realizadas han apuntado exclusivamente hacia simples indicaciones a tener en cuenta, normalmente llevadas a 
cabo dentro de un estudio más general orientado a la aplicación de la filosofía de la gestión de la calidad total en las universidades (Billing, 1996), o a la recomendación de considerar un conjunto de buenas prácticas en los procesos de admisión, que recuerdan al ciclo PDCA (Deming,1986), como las apuntadas por el informe del College Board (2002) citado por Santelices (2007), o las referidas por Rigol (2003) en un estudio detallado de los procesos de admisión de las instituciones de educación superior de los Estados Unidos de América.

Según Rigol (2003), el proceso de admisión debe perseguir maximizar la probabilidad de éxito del alumno, constituyendo como requisito que los procesos de admisión sean capaces de identificar aquellos candidatos con mayor afinidad al perfil de alumno deseado. Así, por ejemplo, si del perfil de alumno deseado se deduce que es requerido un tipo de alumno con determinadas competencias, aptitudes o características, el proceso de admisión debería estar definido de suerte que pueda ser capaz de detectarlas en el momento de la admisión, y, en consecuencia, el orden de prelación utilizado para la admisión de candidatos deberá establecerse tomando en cuenta suficientemente aquella prueba $-\mathrm{o}$ pruebas- de conocimientos o aptitudes orientadas a poder determinarlas. Enfocando los procesos de admisión de esta forma se estaría ayudando a promover el traslado de resultados positivos sobre los grupos de interés, al maximizar la probabilidad de éxito académico de los estudiantes (principal cliente de las universidades), en línea con lo señalado por el subcriterio 6a del modelo EFQM de excelencia (European Foundation for Quality Management, 2013).

Es preciso, sin embargo, señalar que el enfoque propuesto para el estudio del problema no incide sobre la totalidad de las distintos criterios del modelo EFQM, sino exclusivamente con aspectos vinculados con los agentes facilitadores "procesos" y "estrategia" y con los resultados en clientes. Esto es, no se aborda en el presente trabajo el impacto que el diseño específico de los procesos de admisión puede tener sobre otras dimensiones del modelo, como pueden ser los resultados trasladados a la sociedad o sobre los resultados clave de la organización.

Con estos antecedentes se plantea el interés en contrastar si diseñar de manera específica los procesos de admisión de alumnos para determinar la admisión de éstos (alumnos admitidos, pasando a formar parte del grupo de clientes principales de la universidad) tiene relación con su satisfacción, así como sobre su percepción en términos de reputación e imagen de la universidad. En caso de confirmarse, se estaría ayudando a promover el concepto fundamental de excelencia añadir valor para los clientes según el modelo EFQM (European Foundation for Quality Management, 2013). Para ello, este trabajo ha planteado una investigación sobre estudiantes de nuevo ingreso de una universidad de naturaleza privada con experiencia contrastada en el diseño específico de su proceso de admisión, la Universidad Pontificia Comillas.

La página web de la citada universidad informa de que el proceso de admisión en los programas de Grado ofertados por esta institución consideran para el ingreso de estudiantes, además del cumplimiento de los requisitos legales de acceso, criterios de admisión específicos que dependen del programa de estudios solicitado (Universidad Pontificia Comillas, 2015). La referida institución desarrolla un conjunto de pruebas de conocimientos que permite realizar una selección específica para cada programa de estudios; entre los criterios de admisión utilizados se encuentran: la ponderación del expediente académico preuniversitario del candidato y la realización de pruebas de 
conocimientos realizadas en la Facultad/Escuela correspondiente. En resumen, tras formalizar la solicitud por parte del candidato, éste ha de presentarse a pruebas de admisión organizadas por el centro al que esté adscrito el programa de estudios solicitado, y del resultado de las mismas y de la valoración de su expediente académico preuniversitario se determina una nota de corte que establece el orden de prelación para concurrir al ingreso en el programa solicitado.

En consecuencia, a partir del estudio del caso de una universidad privada con experiencia real en el tipo de diseño de procesos de admisión que podrá llevarse a cabo con carácter general por todas las universidades españolas a partir del curso académico 2017/18, tomando como apoyo la estructura del modelo EFQM de excelencia, el objetivo del presente trabajo es investigar si disponer de un proceso específico de admisión de alumnos para determinar la admisión de éstos, tiene relación con la gestión de la calidad en las universidades respecto de sus estudiantes mediante: 1) la satisfacción de aquéllos, y 2) la percepción en términos de reputación e imagen de la universidad que los estudiantes tienen sobre ella. Adicionalmente, se ha abordado el estudio del objetivo planteado considerando la influencia de ciertos factores, entre otros, sexo y forma de acceso al nivel universitario.

\section{Método}

\subsection{Muestra}

La muestra considerada para el estudio ha estado conformada por el conjunto de alumnos de nuevo ingreso matriculados en el curso académico 2014/15 del Grado en Ingeniería Electromecánica (ICAI) y del Grado en Administración y Dirección de Empresas (ADE), organizados respectivamente por la Escuela Técnica Superior de Ingeniería y la Facultad de Ciencias Económicas y Empresariales de la Universidad Pontificia Comillas. El motivo de haber trabajado con dos conjuntos de alumnos ha sido tratar de proporcionar mayor validez al estudio.

La justificación para la elección de los citados Grados es la siguiente, en el caso de ADE, dichos estudios se encuentran entre los que mayor demanda tienen dentro del suE (García, Estellés y Pérez, 2013), además de que cuentan con una amplia presencia en la oferta universitaria de las universidades españolas, 68 de aquéllas ( $82 \%$ del total) ofertan estudios en ADE. En el caso del Grado en Ingeniería Electromecánica, en tanto que estudios pertenecientes a la rama de conocimiento de Ingeniería, los procesos de admisión adquieren mayor importancia si cabe, dada la particularidad que se produce en estos estudios, donde tradicionalmente se han registrado las mayores tasas de abandono en primer curso (Cabrera et al., 2006).

Asimismo, se señala que en la muestra considerada de ADE e ICAI la demanda es notablemente superior a la oferta de plazas, con una nota media de expedientes preuniversitarios por los alumnos admitidos en ADE de 7,78 (sobre diez), y de 8,11 en ICAI.

\subsection{Instrumentos}

Ha sido utilizada una encuesta específica con escala likert de 8 niveles, número par de niveles con el propósito de que los encuestados se posicionasen en sus respuestas en uno u otro sentido. El diseño de la encuesta fue similar para los alumnos de ambos Grados, 
excepto por la particularidad del itinerario o modalidad cursado en cada uno de ellos. En la tabla 1 se presentan las variables de control utilizadas.

Tabla 1. Variables de control de la encuesta

\begin{tabular}{ll}
\hline \multicolumn{1}{c}{ VARIABLE DE CONTROL } & \multicolumn{1}{c}{ PosiBLES VALORES } \\
\hline 1. Año de nacimiento & Edad del estudiante. \\
\hline 2. Sexo del estudiante & Hombre/Mujer. \\
& Bachillerato Español + Prueba de Acceso a la \\
& Universidad (PAU). \\
& Bachillerato Internacional, Europeo, o estudios \\
& de Estados miembros de la Unión Europea, u \\
& otros Estados con los que España haya suscrito \\
& acuerdos internacionales. \\
& Estudios extranjeros obtenidos o realizados en \\
3. Forma de acceso al nivel universitario & Sistemas educativos de Estados ajenos a la \\
& Unión Europea. \\
& Otros.
\end{tabular}

4. Primer curso en el que se realizan los estudios matriculados

5. Itinerario cursado
$\mathrm{Si} / \mathrm{No}$

Alumnos de ICAI: nacional/internacional; alumnos de ADE: ordinario/bilingüe.

Notas: Las variables 1 y 4 fueron utilizadas para evitar considerar encuestas realizadas por alumnos que no fuesen de nuevo ingreso, dado que el recuerdo del proceso de admisión podría haberse visto diluido por el paso del tiempo. Dentro de los estudios de ADE se puede estar cursando la modalidad ordinaria o la referida como ADE bilingüe, donde al menos el $75 \%$ de los créditos se imparten en inglés. Los estudios de ICAI pueden ser cursados en un itinerario nacional o internacional; en este segundo itinerario (de mayor demanda) el estudiante desarrolla, al menos, un año de sus estudios en una universidad socia del extranjero. Tanto la solicitud, como el correspondiente orden de prelación, consideran de forma diferenciada cada uno de los itinerarios o modalidades posibles.

Fuente: Elaboración propia.

La encuesta solicitó la valoración de distintas cuestiones, recogidas en la tabla 2, cuya cumplimentación permitía dar cuenta del valor añadido percibido por el alumno de nuevo ingreso de la Universidad estudiada en los términos descritos en el objetivo de la presente investigación. Conforme al subcriterio 6a del modelo EFQM se solicitaron valoraciones de la percepción en términos de satisfacción con, y reputación e imagen de, la organización en referencia al alcance del objetivo de la investigación.

\subsection{Procedimiento}

Con el propósito de mejorar la herramienta de medida, se distribuyó a una muestra aleatoria de alumnos una primera versión de la encuesta solicitando una respuesta crítica a los ítems planteados en la misma, sirviendo también de pre-test.

La encuesta final fue llevada a cabo coincidiendo con el comienzo del segundo semestre del curso académico 2014/2015. Se distribuyó en las propias aulas, por los autores del presente trabajo, ayudados por los tutores de los distintos grupos, a los alumnos de primer curso de los Grados estudiados (con independencia de la modalidad o itinerario cursado). 
Tabla 2. Variables de control de la encuesta

Exprese su nivel de acuerdo con las siguientes afirmaciones, siendo 1 "en desacuerdo" y 8 "de acuerdo" con las siguientes afirmaciones:

a) Las pruebas de admisión permiten valorar adecuadamente los conocimientos académicos previos antes del ingreso en la Universidad

b) El nivel de exigencia de las pruebas de admisión es superior al de la PAU en su fase general

c) Si bien esta pregunta no está directamente relacionada con el estudio del constructo asociado al objetivo de la investigación, se consideró adecuado medir este aspecto para apuntar si esta cuestión habría de ser un aspecto a tener en cuenta en caso de presentar diferencias significativas, positiva o negativamente

d) La realización de las pruebas de admisión constituye un aspecto diferencial positivo de esta universidad respecto de otras

e) La realización de las pruebas de admisión mejora la reputación de la Facultad/Escuela en la que está matriculado

Exprese su nivel de acuerdo con la siguiente afirmación, siendo 1 bajo nivel de satisfacción y 8 alto nivel de satisfacción:

¿Cuál es su nivel de satisfacción con el procedimiento de admisión de la Facultad o Escuela en la que está matriculado?

Fuente: Elaboración propia.

El número de total de encuestas respondidas fue de 467. Según se recoge en la tabla 3 , el porcentaje de respuesta fue prácticamente del $80 \%$ del total de alumnos matriculados en los referidos estudios.

Tabla 3. Distribución de encuestados

\begin{tabular}{|c|c|c|c|c|}
\hline \multirow{2}{*}{$\begin{array}{c}\text { TIPO DE } \\
\text { ENCUESTA }\end{array}$} & \multicolumn{2}{|c|}{$\begin{array}{c}\text { ¿PRIMER AÑO EN LOS ESTUdIOS } \\
\text { CURSADOS? }\end{array}$} & \multirow{2}{*}{$\begin{array}{c}\text { ALUMNOS DE } \\
\text { NUEVO INGRESO } \\
\text { EN EL PLAN DE } \\
\text { ESTUDIOS (B) }\end{array}$} & \multirow{2}{*}{$\begin{array}{c}\text { PORCENTAJE } \\
\text { REPRESENTADO } \\
\text { A / B }\end{array}$} \\
\hline & No & SI & & \\
\hline $\mathrm{ADE}$ & 9 & 167 & 227 & $73,57 \%$ \\
\hline ICAI & 17 & 274 & 326 & $84,05 \%$ \\
\hline Total & 26 & 441 & 553 & $79,75 \%$ \\
\hline
\end{tabular}

Fuente: Elaboración propia.

Para todas las variables se obtuvieron estadísticos, con su correspondiente distribución de frecuencias. Con el objeto de estudiar si la apreciación global sobre cada variable resultaba indiferente entre los estudiantes, se procedió a realizar los correspondientes contrastes de hipótesis con la prueba $\mathrm{T}$ de un valor, considerando como hipótesis nula: el valor medio de la variable es 4,5 (punto medio de la escala utilizada), frente la hipótesis alternativa que consideraba dicho valor medio distinto. En todos los casos, los contrastes utilizados han considerado un nivel de significación del 5\%.

En todas las variables recogidas se ha estudiado, mediante la prueba $\mathrm{T}$ para la igualdad de medias y tomando en consideración el valor de la prueba de Levene para la igualdad de varianzas, si se presentaban diferencias estadísticamente significativas en función de las variables de control: sexo, forma de acceso al nivel universitario (Bachiller + PAU u otro), plan de estudios cursado (ICAI o ADE) o modalidad o itinerario cursado dentro del Grado. Dadas las características de la muestra, el grupo "otros" incluyó mayoritariamente estudiantes que accedieron al SUE tras haber cursado estudios de Bachillerato Internacional o realizado sus estudios de acceso en un sistema educativo extranjero. 


\section{Resultados}

En primer lugar se señala que realizado el correspondiente análisis de fiabilidad sobre los resultados obtenidos mediante el cuestionario utilizado, el valor obtenido para el alfa de Cronbach fue de 0,73. Según Campo-Arias (2006), este valor es indicativo de un alto grado de consistencia interna en el referido cuestionario. Aspecto que permite dar cuenta de la fiabilidad del constructo: valor añadido percibido por el alumno de nuevo ingreso de la Universidad Pontificia Comillas en los términos descritos en el objetivo de este trabajo.

De todas las variables planteadas, la afirmación: "la realización de las pruebas de admisión mejora la reputación de la Facultad/Escuela en la que está matriculado" presentó la mayor valoración $(6,3)$. Según describe la tabla 4, es significativo que la media de todas las valoraciones obtenidas haya sido superior al punto medio del intervalo $(4,5)$ utilizado para valorar cada afirmación. A continuación, se presentan los resultados obtenidos del estudio individualizado de cada una de las variables obtenidas.

Tabla 4. Estadísticos del valor proporcionado

\begin{tabular}{|c|c|c|c|c|}
\hline & $\mathbf{N}$ & MEDIA & $\begin{array}{c}\text { DESV. } \\
\text { TÍP. }\end{array}$ & $\begin{array}{c}\text { SIG. } \\
\text { (BILAT.) }\end{array}$ \\
\hline $\begin{array}{l}\text { Las pruebas de admisión permiten valorar adecuadamente } \\
\text { los conocimientos académicos previos antes del ingreso } \\
\text { en la Universidad }\end{array}$ & 429 & 5,32 & 1,65 & 0,000 \\
\hline $\begin{array}{l}\text { El nivel de exigencia de las pruebas de admisión es } \\
\text { superior al de la PAU (fase general) }\end{array}$ & 408 & 4,98 & 2,02 & 0,000 \\
\hline $\begin{array}{l}\text { La realización de las pruebas de admisión constituye un } \\
\text { aspecto diferencial positivo de esta universidad respecto } \\
\text { de otras }\end{array}$ & 427 & 6,2 & 1,58 & 0,000 \\
\hline $\begin{array}{l}\text { La realización de las pruebas de admisión mejora la } \\
\text { reputación de la Facultad/Escuela en la que está } \\
\text { matriculado }\end{array}$ & 429 & 6,3 & 1,59 & 0,000 \\
\hline $\begin{array}{l}\text { ¿Cuál es su nivel de satisfacción con el procedimiento de } \\
\text { admisión de la Facultad/Escuela en la que está } \\
\text { matriculado? }\end{array}$ & 430 & 6,12 & 1,37 & 0,000 \\
\hline
\end{tabular}

Así, en relación a la valoración realizada acerca de si las pruebas de admisión de la institución permiten valorar adecuadamente los conocimientos académicos previos antes del ingreso en la Universidad, tal y como se presenta en la figura 1, se han presentado evidencias para afirmar que los alumnos consultados consideran que las pruebas de admisión de la institución investigada permiten valorar adecuadamente los conocimientos académicos previos al ingreso en los estudios.

Al estudiar la valoración en función del tipo de pruebas realizadas según el Grado cursado, se puede afirmar que se han encontrado diferencias entre la valoración realizada por los alumnos de uno y otro centro. Según recoge la tabla 5 , los alumnos de ICAI valoran de forma superior $(5,57)$ la adecuación de las pruebas de admisión a su propósito respecto de los alumnos de $\operatorname{ADE}(4,87)$. Estos resultados pueden sugerir que en el primer caso (ICAI) es más determinante inferir los conocimientos previos de determinadas materias -o competencias desarrolladas-, vinculadas con el ámbito de la ingeniería, para el posterior desarrollo de los estudios universitarios de lo que pueda suceder en el 
segundo caso (ADE), donde quizás tenga más relevancia una formación previa más generalista.

(8). De acuerdo máximo +4

(7). De acuerdo +3

(6). De acuerdo +2

(5). De acuerdo ligero +1

(4). En desacuerdo ligero -1

(3). En desacuerdo -2

(2). En desacuerdo -3

(1). En desacuerdo máximo -4

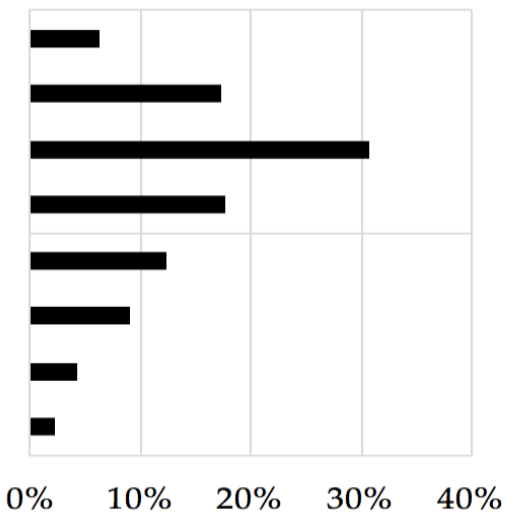

Figura 1. Distribución de la valoración realizada a si las pruebas de admisión de la Universidad Pontificia Comillas permiten valorar adecuadamente los conocimientos académicos previos antes del ingreso en la Universidad

Nota: Media $=5,32$, Desv. Típica $=1,65$, Sig. Prueba T vs 4,5 =,000, $\mathrm{N}=429$.

Fuente: Elaboración propia.

Analizando los datos en función de la forma de acceso al SUE, se han encontrado diferencias entre quienes accedieron por la realización de bachillerato y superación de la PAU respecto de quienes accedieron por otra forma de acceso. Los alumnos del primer grupo evaluaron de forma superior $(5,46)$ la función de las pruebas de admisión de la Universidad Pontificia Comillas, como sistema para valorar adecuadamente los conocimientos académicos previos antes del ingreso en la Universidad, respecto de quienes han accedido por otra forma de acceso $(4,22)$. No se han encontrado diferencias significativas acerca de la cuestión consultada en función del sexo del estudiante, o de la modalidad o itinerario cursado en los estudios.

Tabla 5. Estadísticos de la valoración realizada a si las pruebas de admisión de la Universidad Pontificia Comillas permiten valorar adecuadamente los conocimientos académicos previos antes del ingreso en la Universidad

\begin{tabular}{lllll}
\hline & & N & MEDIA & SIG. \\
\hline \multirow{2}{*}{ Tipo Encuesta } & ADE & 157 & 4,87 & \multirow{2}{*}{$0,000^{*}$} \\
\hline \multirow{2}{*}{ La forma de acceso al sUE es Bachiller + PAU } & ICAI & 272 & 5,57 & \\
& Si & 50 & 4,22 & \multirow{2}{*}{0,000} \\
\hline \multirow{2}{*}{ Sexo del estudiante } & Hombre & 379 & 5,46 & \\
\hline \multirow{2}{*}{ Plan de estudios cursado dentro de ADE } & Mujer & 282 & 5,41 & \multirow{2}{*}{0,116} \\
\hline \multirow{2}{*}{ Plan de estudios cursado dentro de ICAI } & ADE & 146 & 5,14 & \\
& ADE Bilingüe & 69 & 4,97 & \multirow{2}{*}{0,646} \\
\hline
\end{tabular}

Nota: * Nivel de significación obtenido no asumiendo igualdad de varianzas, tras la consideración del resultado de la prueba de Levene.

Fuente: Elaboración propia. 
Otro de los aspectos que ha interesado ser medido es la dificultad percibida de las pruebas de admisión realizadas por la institución, pues aquélla podía influir en la satisfacción de los estudiantes en relación a la forma de decidir su admisión. Tal y como se presenta en la figura 2, la percepción de la dificultad que tienen las pruebas de admisión de la universidad estudiada es superior a la PAU en su fase general.

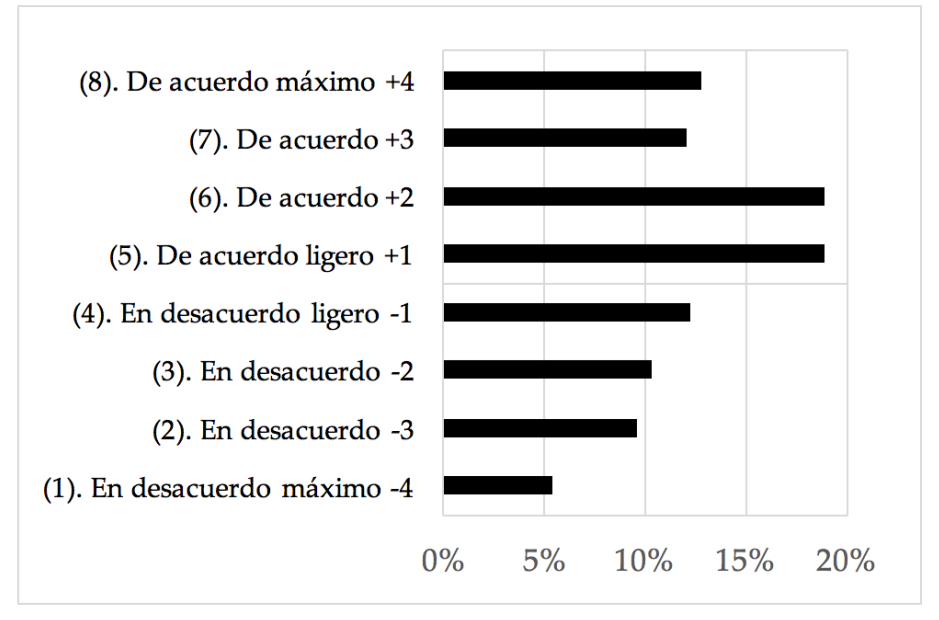

Figura 2. Distribución de la valoración realizada a si las pruebas de admisión de la

Universidad Pontificia Comillas permiten valorar adecuadamente los conocimientos académicos previos antes del ingreso en la Universidad Nota: Media = 4,98, Desv. Típica =2,02, Sig. Prueba T vs 4,5 =,000, $\mathrm{N}=408$. Fuente: Elaboración propia.

En el estudio de la valoración realizada acerca del nivel de exigencia percibido de las citadas pruebas en función de las variables de control, se han encontrado diferencias entre los alumnos del plan de estudios de ICAI según el itinerario cursado (nacional o internacional). Tal y como se presenta en la tabla 6, quienes cursaron el itinerario internacional, itinerario con mayor demanda por parte de solicitantes, consideraron que la dificultad de las pruebas de admisión es similar al nivel de dificultad de la PAU en su fase general, mientras que quienes cursan el itinerario nacional sí perciben una mayor dificultad en las pruebas de admisión. Este aspecto podría señalar la existencia de un diseño de pruebas de admisión más adecuado para concurrir, dentro de ICAI, al itinerario internacional que al nacional.

En cambio, no han sido halladas diferencias en la valoración realizada a esta afirmación en función del Grado cursado, la forma de acceso al SUE o el sexo del estudiante.

Por otro lado, cuando se ha estudiado la relación entre la realización de las pruebas de admisión y la consideración de que ello constituya un aspecto diferencial positivo respecto de otras universidades, según recoge la figura 3, se ha presentado consenso entre los alumnos de los Grados consultados al considerar un aspecto diferencial positivo la realización de pruebas de admisión. Es significativo en este caso que no se han encontrado diferencias en la valoración de esta cuestión en función del Grado o itinerario específico cursado dentro de los estudios, la forma de acceso al SUE o el sexo del estudiante. Este aspecto da cuenta del alto nivel de homogeneidad al respecto por parte de la muestra considerada. 
Tabla 6. Estadísticos de la valoración realizada acerca de si el nivel de exigencia de las pruebas de admisión de la Universidad Pontificia Comillas es superior al de la PAU (fase general)

\begin{tabular}{llrrr}
\hline & & N & MEDIA & SIG. \\
\hline \multirow{2}{*}{ Tipo Encuesta } & ADE & 141 & 5,09 & \multirow{2}{*}{0,417} \\
\hline \multirow{2}{*}{ La forma de acceso al SUE es Bachiller + PAU } & ICAI & 267 & 4,92 & \\
\hline \multirow{2}{*}{ Sexo del estudiante } & $\mathrm{Si}$ & 29 & 5,03 & \multirow{2}{*}{0,881} \\
\hline \multirow{2}{*}{ Plan de estudios cursado dentro de ADE } & Hombre & 379 & 4,98 & \\
& Mujer & 271 & 4,99 & \multirow{2}{*}{0,877} \\
\hline \multirow{2}{*}{ Plan de estudios cursado dentro de ICAI } & ADE & 136 & 4,96 & \\
& ADE Bilingüe & 68 & 5,07 & \multirow{2}{*}{0,993} \\
\hline Funternal & Internacional & 77 & 5,07 & \\
\hline
\end{tabular}

Fuente: Elaboración propia.

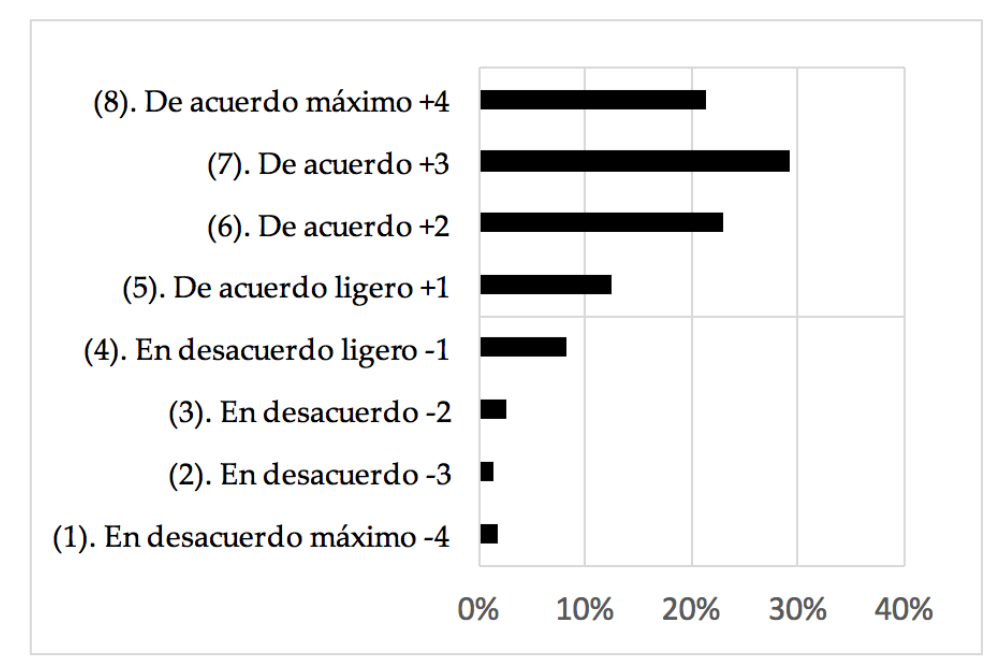

Figura 3. Distribución de la valoración realizada acerca de si la realización de las pruebas de admisión en la Universidad Pontificia Comillas constituye un aspecto diferencial positivo respecto de otras universidades

Nota: Media $=6,2$, Desv. Típica $=1,58$, Sig. Prueba T vs 4,5 =,000, N =427.

Fuente: Elaboración propia.

Tal y como se apuntaba al principio del epígrafe, al analizar la evaluación realizada acerca de si la realización de pruebas de admisión mejora la reputación de la Facultad/Escuela en la que se está matriculado, se han obtenido los mayores porcentajes de consenso en la encuesta realizada. Según se puede observar en la figura 4, la valoración media obtenida a la consulta realizada es de 6,3. 
(8). De acuerdo máximo +4

(7). De acuerdo +3

(6). De acuerdo +2

(5). De acuerdo ligero +1

(4). En desacuerdo ligero -1

(3). En desacuerdo -2

(2). En desacuerdo -3

(1). En desacuerdo máximo -4
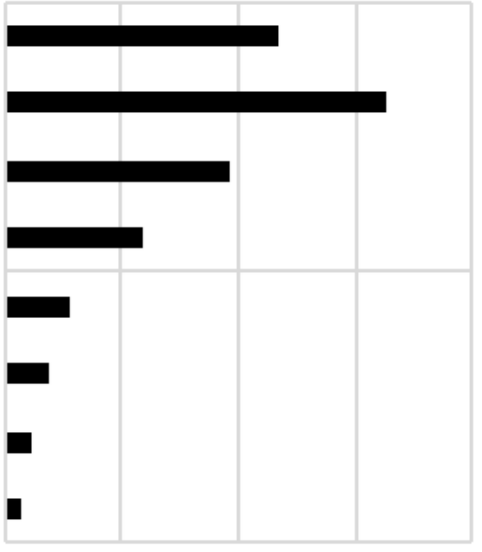

$0 \% \quad 10 \% \quad 20 \% \quad 30 \% \quad 40 \%$

Figura 4. Distribución de la valoración realizada acerca de si la realización de las pruebas de admisión en la Universidad Pontificia Comillas constituye un aspecto diferencial positivo respecto de otras universidades

Nota: Media =6,3, Desv. Típica =1,59, Sig. Prueba T vs 4,5=,000, N =429.

Fuente: Elaboración propia.

En la tabla 7 se describe que los alumnos encuestados manifiestan un consenso mayoritario en señalar que la realización de las pruebas de admisión mejora la reputación de la Facultad/Escuela. En función de la forma de acceso al suE, la valoración es superior para quienes han accedido por tener el título de bachiller español y haber superado la PAU $(6,35)$ que la de aquéllos que han accedido por otra forma de acceso $(5,86)$, donde fundamentalmente se agrupan alumnos procedentes de sistemas educativos extranjeros. Estos resultados apuntarían a una oportunidad dentro de España para diferenciar a la universidad que realice pruebas específicas de admisión en términos de reputación.

Tabla 7. Estadísticos de la valoración realizada acerca de si la realización de pruebas de admisión mejora la reputación de la Facultad/Escuela en la que se está matriculado

\begin{tabular}{llrrrr}
\hline & & N & MEDIA & SIG. \\
\hline \multirow{2}{*}{ Tipo Encuesta } & ADE & 157 & 6,38 & \multirow{2}{*}{0,396} \\
\hline \multirow{2}{*}{ La forma de acceso al SUE es Bachiller + PAU } & ICAI & 272 & 6,25 & \\
& Si & 50 & 5,86 & \multirow{2}{*}{0,039} \\
\hline \multirow{2}{*}{ Sexo del estudiante } & Hombre & 379 & 6,35 & \\
\hline \multirow{2}{*}{ Plan de estudios cursado dentro de ADE } & Mujer & 281 & 6,26 & \multirow{2}{*}{0,550} \\
& ADE & 147 & 6,36 & \\
\hline \multirow{2}{*}{ Plan de estudios cursado dentro de ICAI } & ADE Bilingüe & 69 & 6,58 & \multirow{2}{*}{0,141} \\
& Internacional & 193 & 6,09 & \\
\hline
\end{tabular}

Fuente: Elaboración propia.

En cambio, no se han presentado diferencias en la valoración obtenida acerca de si la realización de las pruebas de admisión mejora la reputación de la Facultad/Escuela en 
función de los estudios cursados, así como dentro de los posibles itinerarios cursados en los referidos Grados. Tampoco existen diferencias en la valoración realizada en función del sexo de los alumnos.

Por su parte, al estudiar la relación con el nivel de satisfacción de los encuestados con el procedimiento específico de admisión que sigue la Universidad investigada, según se muestra en la figura 5, un porcentaje mayoritario de respuestas corresponde con una valoración alta. La media se ha situado en 6,12 y apunta que es posible obtener valoraciones de niveles de satisfacción altos con procesos específicos de admisión.

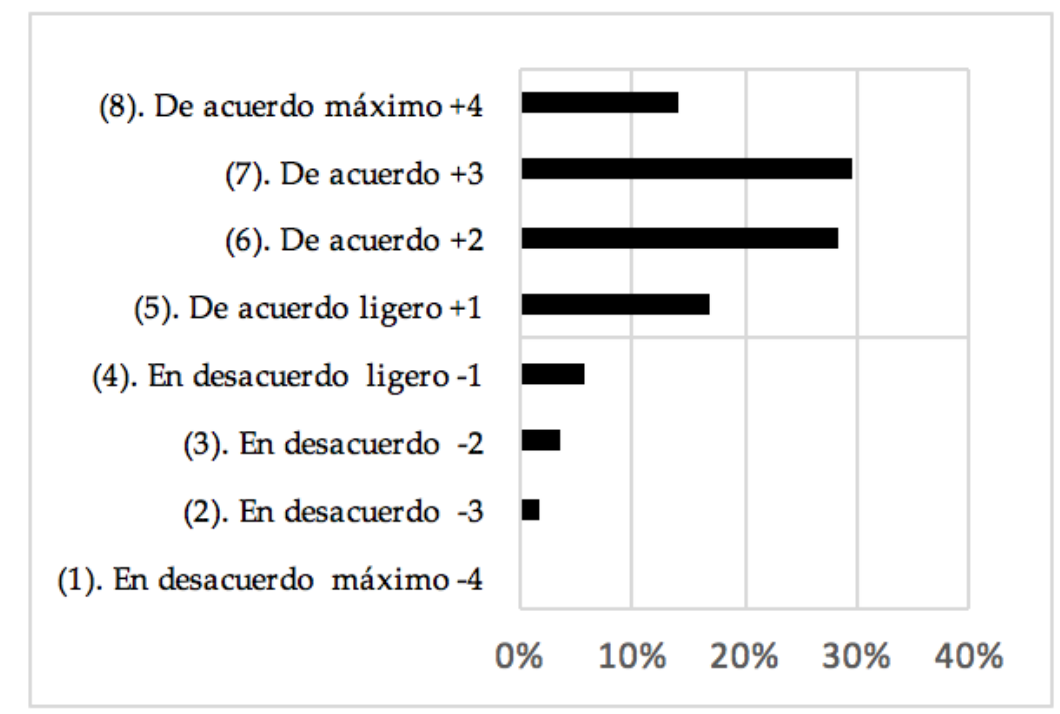

Figura 5. Distribución de la valoración realizada acerca de si la realización de las pruebas de admisión en la Universidad Pontificia Comillas constituye un aspecto diferencial positivo respecto de otras universidades

Nota: Media $=6,12$, Desv. Típica $=1,37$, Sig. Prueba T vs 4,5 =,000, $\mathrm{N}=430$.

Fuente: Elaboración propia.

Además, cuando se ha estudiado la valoración del nivel de satisfacción manifestado con el procedimiento de admisión de la Facultad/Escuela en la que han ingresado los encuestados, se han encontrado diferencias en función de la forma de acceso al SUE. Tal y como presenta la tabla 8 , quienes han obtenido el título de bachiller español y superado la PAU valoran de forma superior $(6,17)$ el procedimiento de admisión de la Facultad/Escuela en la que están matriculados que los estudiantes que accedieron con otra forma de acceso (5,71). De nuevo, se apunta la posibilidad de diferenciar positivamente, dentro de España, aquella universidad que realice pruebas específicas de admisión.

De forma particular, dentro del colectivo de alumnos de ICAI, la valoración del nivel de satisfacción manifestado con el procedimiento de admisión de la Facultad/Escuela, en función del itinerario cursado presenta diferencias. Los alumnos que cursan el itinerario internacional manifiestan una valoración superior $(6,50)$ de su satisfacción con el procedimiento de admisión respecto de aquellos que cursan el itinerario nacional $(6,09)$. Este aspecto podría estar relacionado con una mejor adecuación del procedimiento de admisión realizado para concurrir al proceso de admisión del itinerario internacional en los estudios de ICAI, respecto del itinerario nacional. 
No se han hallado diferencias en función de la modalidad cursada dentro de este último grado, ni del sexo del alumno.

Tabla 8. Estadísticos de la valoración realizada acerca del nivel de satisfacción con el procedimiento de admisión de la Facultad/Escuela en la que se está matriculado

\begin{tabular}{|c|c|c|c|c|}
\hline & & $\mathbf{N}$ & MEDIA & SIG. \\
\hline \multirow{2}{*}{ Tipo Encuesta } & $\mathrm{ADE}$ & 156 & 5,96 & \multirow{2}{*}{0,077} \\
\hline & ICAI & 274 & 6,2 & \\
\hline \multirow{2}{*}{ La forma de acceso al SUE es Bachiller + PAU } & No & 51 & 5,71 & \multirow{2}{*}{0,023} \\
\hline & $\mathrm{Si}$ & 379 & 6,17 & \\
\hline \multirow{2}{*}{ Sexo del estudiante } & Hombre & 281 & 6,05 & \multirow{2}{*}{0,173} \\
\hline & Mujer & 148 & 6,24 & \\
\hline \multirow{2}{*}{ Plan de estudios cursado dentro de ADE } & $\mathrm{ADE}$ & 68 & 5,97 & \multirow{2}{*}{0,831} \\
\hline & ADE Bilingüe & 46 & 5,91 & \\
\hline \multirow{2}{*}{ Plan de estudios cursado dentro de ICAI } & Internacional & 78 & 6,50 & \multirow{2}{*}{0,023} \\
\hline & Nacional & 196 & 6,09 & \\
\hline
\end{tabular}

Fuente: Elaboración propia.

\section{Discusión y conclusiones}

A partir del estudio de caso de una universidad privada, este trabajo ha planteado el propósito de investigar si, con las oportunidades que ha incorporado recientemente la legislación vigente, diseñar de forma específica los procesos de admisión para determinar el ingreso de alumnos ayudaría a promover la calidad en las universidades españolas entre sus estudiantes. Ello se plantea con el propósito último de avanzar en el conocimiento en el campo de la promoción de la gestión de la calidad en las universidades.

Las principales conclusiones que se han obtenido en el presente estudio han sido las siguientes:

- Se presentan evidencias que señalan que un diseño específico permite valorar adecuadamente los conocimientos académicos previos de los solicitantes antes del ingreso en la Universidad.

- El nivel de exigencia de pruebas específicas de conocimientos/competencias puede ser percibido de forma distinta al nivel de exigencia de la actual PAU española. Este aspecto habría de ser atendido especialmente en el diseño específico de los procesos de admisión de Grado.

- La realización de pruebas específicas de conocimientos, competencias y/o aptitudes puede promover la constitución de un aspecto diferencial, positiva o negativamente, respecto de otras universidades.

- La realización de pruebas de admisión puede promover una mejora de la reputación percibida por los estudiantes de la Facultad/Escuela que las realice.

- Un diseño específico de los procesos de admisión no implica que exista disconformidad o perjuicio en el nivel de satisfacción de los alumnos de nuevo ingreso con el procedimiento de admisión de la Facultad/Escuela que lo lleve a cabo; puede producirse lo contrario al constituirse como un aspecto diferencial positivo de la Facultad/Escuela. 
- El diseño específico de los procesos de admisión ha de tomar en consideración el impacto en el nivel de satisfacción que puede conllevar sobre alumnos que proceden de sistemas educativos extranjeros, incluyendo a los alumnos que han cursado el Bachillerato Internacional. Los resultados obtenidos apuntarían a la necesidad de profundizar sobre la adecuación del procedimiento de admisión al referido colectivo de solicitantes.

Los puntos 3, 4 y 5 apuntarían a la consideración de fortalezas del modelo de diseño específico de procesos de admisión, mientras que el 6 podría indicar una debilidad. No obstante, para poner en valor el modelo planteado respecto de un modelo de diseño único, como el establecido hasta el curso académico 2017/18 para alumnos procedentes del título de Bachiller español, habría que considerar otras cuestiones (entre otras, costes económicos para las universidades y para los estudiantes, cuestiones del reconocimiento o no de la validez de pruebas entre distintas universidades, o posibles incompatibilidades de fechas) en una investigación ad-hoc.

Sin perjuicio de lo anterior, los resultados obtenidos sugieren que un diseño específico de los procesos de admisión de alumnos en las universidades españolas puede ser gestionado de manera que promueva la satisfacción de los estudiantes en cuanto a la forma de determinar la admisión de alumnos por parte de la universidad, así como que sirvan para mejorar la percepción en términos de reputación e imagen de la universidad que los estudiantes tienen sobre ella, incentivándose por ello, según el modelo EFQM, avanzar en la mejora de la gestión de la calidad basada en la excelencia dentro de la organización. Estos resultados estarían alineados con lo señalado en los trabajos previos de Rigol (2003) y Santelices (2007), en referencia a otros sistemas educativos.

No obstante, es preciso señalar que las limitaciones de este trabajo no son menores y deben de ser tenidas en cuenta para la generalización de los resultados:

- La naturaleza privada de la universidad y las características del plan de estudios donde se ha llevado a cabo la investigación. Por un lado, se ha podido presentar un posible exceso de homogeneidad en el perfil del alumno consultado, aspecto que podría haber incorporado cierto sesgo en las valoraciones recogidas en un sentido de mayor o menor sensibilidad a las propuestas planteadas. Por otro, el caso planteado parte del supuesto de considerar programas de estudios donde la demanda de solicitudes supera la oferta, aspecto que no se registra en todos los programas ofertados dentro del SUE.

- El establecimiento de criterios específicos de admisión solo actúa sobre una parte del conjunto de agentes facilitadores, los relacionados con la admisión de alumnos (procesos y estrategia), que se desarrollan dentro del tipo de organización que constituye una universidad.

- No se aborda el impacto del enfoque utilizado para gestionar los procesos de admisión sobre los criterios 7, 8 y 9 del modelo EFQM, resultados en las personas de la organización, en la sociedad y resultados clave de la organización. No hacerlo limita la evaluación del impacto real trasladado en términos de gestión de excelencia en la organización. 
Para concluir, se apunta como futura línea de investigación la continuidad del presente trabajo en dos posibles líneas de actuación no inconexas. La primera, profundizar en el estudio de la relación de un diseño específico de los procesos de admisión dentro del conjunto de todos los agentes facilitadores del modelo EFQM, ampliando el análisis considerando del posible impacto de la propuesta en los resultados trasladados en términos de promoción de la excelencia. La segunda, ampliar el estudio recogiendo las particularidades y consideraciones específicas de alumnos de otras titulaciones/ramas de conocimiento del SUE.

\section{Referencias}

Alfaro-Saiz, J. J., Carot-Sierra, J. M., Rodríguez-Rodríguez, R. y Jabaloyes-Vivas, J. M. (2011). Seeking organisational excellence by using the information coming from the EFQM excellence model as starting point: application to a real case. Total Quality Management $\mathbb{E}^{2}$ Business Excellence, 22(8), 853-868. doi:10.1080/14783363.2011.597595

Alonso-Arévalo, J. (2003). Evaluación de bibliotecas universitarias con el modelo EFQM. Recuperado de http://hdl.handle.net/10366/83062

Antón, A. M. y Castilla, J. I. M. (junio, 2010). El modelo EFQM aplicado a la gestión de la Fundación General de la Universidad Politécnica de Madrid. Utilización para la evaluación de proyectos de cooperación. Comunicación presentada en el XIV International Congress on Project Engineering. Madrid.

Bandyopadhyay, J. K. y Lichtman, R. (2007). Six sigma approach to quality and productivity improvement in an institution for higher education in the United States. International Journal of Management, 24(4), 804-809.

Billing, D. (1996). Managing quality policy and projects in a university. Total Quality Management, 7(2), 203-212. doi:10.1080/09544129650034954

Cabrera, L., Bethencourt, J. T., Alvarez, P. y González, M. (2006). El problema del abandono de los estudios universitarios. RELIEVE, 12(2), 1-24. doi:10.7203/relieve.12.2.4226

Calvo-Mora, A. y Criado García-Legaz, F. (febrero, 2003). Análisis del poder predictivo del modelo EFQM para la gestión y mejora de la calidad en la universidad pública española. Comunicación presentada en XIII Jornadas Hispano-Lusas de Gestión Científica. Lugo.

Calvo-Mora, A., Leal, A. y Roldán, J. L. (2005). Relationships between the EFQM model criteria: a study in spanish universities. Total Quality Management $\mathscr{E}^{2}$ Business Excellence, 16(6), 741770. doi:10.1080/14783360500077708

Campatelli, G., Citti, P. y Meneghin, A. (2011). Development of a simplified approach based on the EFQM model and six sigma for the implementation of TQM principles in a university administration. Total Quality Management \& Business Excellence, 22(7), 691-704. doi:10.1080/14783363.2011.585755

Campo-Arias, A. (2006). Usos del coeficiente de alfa de Cronbach. Biomedica, 26(4), 585-8. doi:10.7705/biomedica.v26i4.327

Cantón, I. y Vázquez, J. L. (2010). Los procesos en gestión de calidad. Un ejemplo en un centro educativo. REICE. Revista Iberoamericana sobre Calidad, Eficacia y Cambio en Educación, 8(5), 59-68.

Cruickshank, M. (2003). Total quality management in the higher education sector: a literature review from an international and australian perspective. Total Quality Management \& Business Excellence, 14(10), 1159-1 167. doi:10.1080/1478336032000107717 
Dahlgaard, J. J., Chen, C. K., Jang, J. Y., Banegas, L. A. y Dahlgaard-Park, S. M. (2013). Business excellence models: limitations, reflections and further development. Total Quality Management E' Business Excellence, 24(5), 519-538. doi:10.1080/14783363.2012.756745

Dahlgaard-Park, S. M. (2008). Reviewing the European excellence model from a management control view. The TQM Journal, 2O(2), 98-119. doi:10.1108/17542730810857345

Feixas, M. (2004). De Bolonia a Berlín. Revista Interuniversitaria de Formación del Profesorado, $18(1), 149-162$.

García, H. C., Estellés, T. C. y Pérez, F. J. M. (2013). De la licenciatura al grado en administración y dirección de empresas: un análisis comparativo desde las necesidades de las empresas. EDUCADE: Revista de Educación en Contabilidad, Finanzas y Administración de Empresas, 4, 56-76.

Harvey, L. (1998). An assessment of past and current approaches to quality in higher education. Australian Journal of Education, 42(3), 237-255. doi:10.1177/000494419804200303

Koch, J. V. y Fisher, J. L. (1998). Higher education and total quality management. Total Quality Management, 9(8), 659-668. doi:10.1080/0954412988136

Lau, R. S. y Anderson, C. A. (1998). A three-dimensional perspective of total quality management. International Journal of Quality \& Reliability Management, 15(1), 85-98. doi:10.1108/02656719810199277

Marshall, S. J. (1998). Professional development and quality in higher education institutions of the $21 \mathrm{st}$ century. Australian Journal of Education, 42(3), 321-334. doi:10.1177/000494419804200308

Martín, J. I. (2005). La integración de la innovación, el aprendizaje, la dirección del conocimiento y la mejora continua de la calidad del servicio en la administración pública. Administración de Andalucía: Revista Andaluza de Administración Pública, 57, 255-279.

Ministerio de Educación, Cultura y Deporte. (2015). Datos básicos del sistema universitario español. Curso 2014-2015. Recuperado de http://www.mecd.gob.es/educacion-mecd/areaseducacion/universidades/estadisticas-informes/datos-cifras.html

Ololube, N. P. (2006). An approach to quality improvement of education in Nigeria through EFQM excellence model. The African Symposium: An On Line Journal of African Educational Research Network, 6, 7-22.

Osseo-Asare, A. E. y Longbottom, D. (2002). The need for education and training in the use of the EFQM model for quality management in UK higher education institutions. Quality Assurance in Education, 10(1), 26-36. doi:10.1108/09684880210416085

Rigol, G. (2003). Admissions decision-making models: how US institutions of higher education select undergraduate students. Nueva York: The College Board.

Ruiz-Carrillo, J. I. y Fernández-Ortiz, R. (2005). Theoretical foundation of the EFQM model: the resource-based view. Total Quality Management \& Business Excellence, 16(1), 31-55. doi:10.1080/1478336042000309857

Rusjan, B. (2005). Usefulness of the EFQM excellence model: theoretical explanation of some conceptual and methodological issues. Total Quality Management \& Business Excellence, 16(3), 363-380. doi:10.1080/14783360500053972

Santelices, M. V. (2007). Procesos de admisión a instituciones de educación superior en el mundo: antecedentes bibliográficos para la consideración de criterios complementarios en el proceso de admisión a la Pontificia Universidad Católica de Chile. Santiago: Universidad Pontificia Católica de Chile. 
Sila, I. y Ebrahimpour, M. (2002). An investigation of the total quality management survey based research published between 1989 and 2000: a literature review. International Journal of Quality \& Reliability Management, 19(7), 902-970. doi:10.1108/02656710210434801

Srikanthan, G. y Dalrymple, J. F. (2002). Developing a holistic model for quality in higher education. Quality in Higher Education, 8(3), 215-224. doi:10.1080/1353832022000031656

Srikanthan, G. y Dalrymple, J. F. (2005). Implementation of a holistic model for quality in higher education. Quality in Higher Education, 1 1(1), 69-81. doi:10.1080/13538320500077686

Steed, C. (2002). Excellence in higher education: evaluating the implementation of the EFQM excellence model in higher education in the UK. Beiträge zur Hochschulforschung, 24(1), 7499.

Universidad Complutense de Madrid. (2015). Memoria para la verificación del título de grado en medicina por parte de la agencia nacional de evaluación de la calidad y acreditación (ANECA). Recuperado de https://www.ucm.es/data/cont/docs/97-2015-06-07-grado_medicina.pdf

Universidad Pontificia Comillas. (2015). Proceso de admisión. Recuperado de http://www.upcomillas.es/es/admisiones/informacion-general/informacion-de-interes

Vázquez García, J. A. (2015). Nuevos escenarios y tendencias universitarias. Revista de Investigación Educativa, 33(1), 13-26.

Villarruel, M. (2010). Calidad en la educación superior: un análisis reflexivo sobre la gestión de sus procesos en los centros educativos de América. REICE. Revista Iberoamericana sobre Calidad, Eficacia y Cambio en Educación, 8(5), 110-118

Zink, K. J. y Schmidt, A. (1995). Measuring universities against the european quality award. Total Quality Management, 6(5), 547-561. doi:10.1080/09544129550035206

\section{Breve CV de los autores}

\section{José María Ortiz Lozano}

Doctor por la Universidad Pontificia Comillas (ICAI/ICADE), dentro del Programa Oficial de Doctorado en Economía y Empresa; Master en Investigación en Economía y Empresa (ICAI/ICADE); Master in Business Administration Executive (ICAI/ICADE); Licenciado en Investigación y Técnicas de Mercado (ICAI/ICADE); Diplomado en Estadística, Universidad Complutense de Madrid. En la actualidad desarrolla su actividad profesional como Director del Servicio de Gestión Académica y Títulos, Vocal del Comité de Calidad, y Vocal del Comité de Seguimiento de Indicadores y Estadísticas de la Universidad Pontificia Comillas. Como investigador se dedica a la aplicación de sistemas de gestión de calidad en organizaciones educativas y al análisis de datos mediante técnicas multivariantes aplicadas al ámbito de la educación y a la gestión de recursos humanos. ORCID ID: 0000-0002-8317-4393. Email: jmortiz@comillas.edu

\section{Antonio Rúa Vieites}

Profesor Propio Agregado del Departamento de Métodos Cuantitativos de la Facultad de Ciencias Económicas y Empresariales (CC.EE) de la Universidad Pontifica Comillas (Madrid). Licenciado en Ciencias Físicas, Máster en Meteorología Teórica y Aplicada, Doctor en Ciencias Físicas, Licenciado en Ciencias y Técnicas Estadísticas y Executive MBA. Profesor de Estadística, Matemáticas, Investigación Operativa y Análisis de datos 
en diferentes cursos de Grado y Postgrado en el entorno de la Economía, Empresa y Marketing. Como investigador se dedica al Análisis de Datos mediante el uso, fundamentalmente, de técnicas multivariantes, en el ámbito de la educación, administración de empresas, economía y sociología. ORCID ID: 0000-0002-6915-2067. Email: rvieitesicade.comillas.edu 